\title{
MAPEO DE LA INVESTIGACIÓN EN LA GESTIÓN INTERNACIONAL DE LOS RECURSOS HUMANOS
}

\section{MAPPING RESEARCH IN THE INTERNATIONAL HUMAN RESOURCE MANAGEMENT FIELD}

Mónica Santana (Universidad Pablo de Olavide) ${ }^{1}$

Susana Pasamar (Universidad Pablo de Olavide) ${ }^{2}$

\section{Resumen:}

A pesar del interés en la Gestión Internacional de los Recursos Humanos en los últimos años, aún deben abordarse varios problemas para proporcionar claridad y comprensión conceptual. Este artículo tiene como objetivo ofrecer una revisión sistemática de la literatura de la Gestión Internacional de los Recursos Humanos, destacando los principales temas y tendencias observados en este campo. El estudio ofrece una evaluación crítica, profundizando la comprensión de lo que es la Gestión Internacional de los Recursos Humanos, e identificando las líneas de investigación futuras más prometedoras. Se desarrolló un análisis de mapeo científico basado en redes de co-palabras de la literatura, utilizando la herramienta bibliométrica SciMAT. Se siguieron tres pasos: primero, se detectaron temas de investigación; segundo, se visualizaron los temas de investigación y las redes temáticas; y tercero, se midieron mediante análisis de rendimiento las contribuciones hechas por los temas de investigación. Mil setecientos siete documentos publicados entre 1974 y 2020 fueron recuperados de la Web of Science. En el último periodo (2015-2020) los temas motores son el ajuste intercultural, las multinacionales, las carreras globales y la gestión intercultural. La satisfacción de los trabajadores es un tema especializado, mientras que China es un tema transversal. La gestión global del talento, las mujeres en el contexto internacional y la diversidad son temas emergentes. Este análisis ayudará a informar a los investigadores, profesionales y administraciones públicas sobre la importancia de la Gestión Internacional de los Recursos Humanos.

Palabras claves: Gestión Internacional de los Recursos Humanos; GIRRHH; carreras globales; mapeo de la ciencia; SciMAT

Código JEL: M50.

\begin{abstract}
:
\footnotetext{
1 msanher@upo.es, Universidad Pablo de Olavide.

2 spasrey@upo.es, Universidad Pablo de Olavide.

Recibido 24 de marzo de 2020. Aceptado 21 de mayo de 2020.
}

Despite the interest in International Human Resource Management (IHRM) in recent years, several issues still need to be addressed to provide clarity and conceptual understanding. This article aims to offer a systematic review of the literature on IHRM, highlighting the main themes and trends observed in this field. The study offers a critical evaluation, deepening the comprehension of IHRM, and identifying the most promising future lines of research. A scientific mapping analysis based on co-word networks of the literature was developed, using the SciMAT bibliometric tool. Three steps were followed: first, research themes were detected; second, the research themes and thematic networks were visualised; and third, the contributions made by the research topics were measured by performance analysis. One thousand seven hundred and seven published documents between 1974 and 2020 were retrieved from the Web 
of Science. In the last period (2015-2020) the driving themes are cross-cultural adjustment, multinationals, global careers and cross-cultural management. Job satisfaction is a specialised issue, while China is a transversal issue. Global talent management, women in the international context and diversity are emerging themes. This analysis will help inform researchers, professionals and public administrations about the importance of IHRM.

Keywords: International Human Resource Management; IHRM; global careers; bibliometrics; SciMAT.

JEL Code: M50.

\section{INTRODUCCIÓN}

La internacionalización es clave para la supervivencia de las empresas e implica una adaptación y esfuerzo extra de todas las áreas funcionales de la empresa, de las cuales la Gestión Internacional de Recursos Humanos es una de las más relevantes (Bornay-Barrachina, 2019).

En las últimas décadas ha habido un aumento sin precedentes en el número de organizaciones que han internacionalizado sus operaciones, lo que ha supuesto una creciente demanda de talento internacional. En ese sentido, la movilidad internacional ha pasado a ser considerada una parte muy importante del desarrollo de carrera de los empleados (Pasamar y Gallurt, 2019). El desplazamiento de mano de obra a nivel internacional necesario para la expansión de estos negocios ha provocado que los problemas asociados con la Gestión Internacional de los Recursos Humanos a través de las fronteras internacionales sean cada vez más importantes para los profesionales y académicos interesados (De Cieri et al., 2005).

A pesar de las cuatro décadas de investigación intensa relativa a la Gestión Internacional de los Recursos Humanos la literatura académica ha tenido un éxito parcial en la búsqueda de soluciones que pueden trascender las divisiones nacionales, culturales y económicas (Ozbilgin, 2004). Más aún, muchos estudios reclaman la necesidad de estudios sistemáticos que den orden al campo de la Gestión Internacional de los Recursos Humanos, sobre todo teniendo en cuenta que han aparecido nuevas necesidades y contextos diferentes a los tradicionalmente estudiados (Dabic et al., 2015).

Las revisiones sistemáticas y los análisis bibliométricos son herramientas valiosas para abordar la falta de claridad en determinadas áreas de conocimiento. La bibliometría hace referencia a la incorporación de técnicas estadísticas y matemáticas a diferentes fuentes de información (Hood y Wilson, 2001). Son útiles para realizar una revisión sistemática con características dinámicas y estructurales de la investigación científica (Cobo et al., 2011b). El mapeo bibliométrico o el mapeo científico constituye una visualización espacial de cómo se conectan las categorías, los campos, los documentos o los autores (Small, 1999). Los métodos bibliométricos se han utilizado antes en el área de la Gestión de los Recursos Humanos (Santana y Lopez-Cabrales, 2019; Santana et al., 2020). Particularmente, en el campo de la Gestión Internacional de los Recursos Humanos no existe una revisión bibliométrica global con mapeo científico de todo el área, tan solo han surgido estudios más concretos que observan particularmente la problemática de los expatriados (Andersen, 2019), que es tan solo una de las tipologías dentro de los empleados en contextos internacionales (Dickmann et al., 2008).

El objetivo de este artículo es, por lo tanto, contribuir a una mejor comprensión de la Gestión Internacional de los Recursos Humanos, identificando los temas más prometedores dentro de este campo y definiendo tendencias a lo largo del tiempo. El análisis bibliométrico desarrollado en este documento establece el estado del arte de la Gestión Internacional de los 
Recursos Humanos, y señala nuevos temas de investigación de acuerdo con el creciente número de documentos, citas y tasas de impacto en los últimos años. En consecuencia, este estudio ayudará a comunicar a los investigadores, profesionales y administraciones públicas las tendencias más relevantes y los temas de mayor impacto en la Gestión Internacional de los Recursos Humanos.

\section{METODOLOGÍA}

Para realizar este análisis, se utiliza una herramienta de software de análisis de mapeo científico llamada SciMAT (Cobo, López-Herrera, Herrera-Viedma, y Herrera, 2012). Esta herramienta se basa en el enfoque de análisis de mapeo científico presentado por Cobo, LópezHerrera, Herrera-Viedma, y Herrera (2011) que permite realizar estudios de mapeo científico bajo un marco longitudinal. Se expone a continuación una breve descripción de la metodología utilizada (no obstante, para un conocimiento profundo de la herramienta, consulte el trabajo de Cobo y otros (2011a, 2011b, 2012). Para realizar un análisis riguroso, se describe cada paso del proceso. El análisis de mapeo científico sigue estas etapas: búsqueda de datos, refinamiento de datos, estandarización y creación de la red, creación de mapas, análisis y visualización y análisis de rendimiento (figura 1) (Börner et al., 2005; Cobo et al., 2011b).

\begin{tabular}{|c|c|}
\hline FIGURA. 1. ETAPAS DEL MAPEO CIENTÍFICO \\
\hline Búsqueda de datos & $\begin{array}{c}\text { - Selección de palabras clave correspondientes al tópico de la } \\
\text { Gestión internacional RRHH } \\
\text { - Wos, colección principal, 1974-2020 }\end{array}$ \\
\hline $\begin{array}{c}\text { Refinamiento de } \\
\text { datos }\end{array}$ & - Refinado de datos mal escritos o duplicados \\
\hline $\begin{array}{c}\text { Estandarización y } \\
\text { creación de la red }\end{array}$ & $\begin{array}{l}\text { - Creación de la red (co-ocurrencia) y reducción de la red } \\
\text { - Medida de similaridad para estandarizar la red: Indice de } \\
\text { equivalencia }\end{array}$ \\
\hline Creación del mapa & $\begin{array}{c}\text { - Implementar un algoritmo de agrupamiento para obtener la } \\
\text { creación del mapa científico: centros simple }\end{array}$ \\
\hline Análisis y \\
visualización & $\begin{array}{c}\text { - obtención e identificación de los temas de investigación y } \\
\text { redes temáticas: diagramas estratégicos y clústeres }\end{array}$ \\
\hline $\begin{array}{c}\text { Análisis de } \\
\text { resultados }\end{array}$ & $\begin{array}{c}\text { - Medir la contribución de los temas de investigación al campo } \\
\text { cientíico }\end{array}$ \\
\hline - Identificar los sub-campos más prolificos y de mayor impacto \\
\hline Fuente: Elaboración propia.
\end{tabular}

En la primera etapa (búsqueda de datos), se recuperaron documentos de la base de datos de Web of Science (WoS), que de acuerdo con Norris y Oppenheim (2007) ofrece una cobertura exhaustiva de la literatura de ciencias sociales y la mayor cobertura en años (Sánchez et al., 2017). Este estudio incluye todos los artículos y revisiones publicados en WoS en los campos de Business y Management. El período de análisis en este documento fue de 1974 a 2020, siendo 1974 el primer año en que se identificó un documento relacionado con la Gestión Internacional de los Recursos Humanos. Las palabras clave incorporadas en la búsqueda de WoS se obtuvieron con la ayuda de dos reputados catedráticos internacionales en el campo de 
la Gestión Internacional de los Recursos Humanos y observando las palabras clave de los artículos más relevantes en esta área (ver tabla 1).

\title{
TABLA 1. PALABRAS CLAVE USADAS EN EL CAMPO DE LA GESTIÓN INTERNACIONAL DE LOS RECURSOS HUMANOS PARA LA BÚSQUEDA EN WOS
}

\begin{abstract}
$\mathrm{TS}=$ ("IHRM" or "international human resource management" or "international HR management" or "global staffing" or "global talent management" or "international assignments" or ("expatriat*" and "IHRM") or ("expatriat*" and "international human resource management") or "global career*" or "expatriat* manage*" or "cross cultural management" or ("expatriat*" and "HRM") or ("expatriat*" and "human resource management") or ("global mobility" and "IHRM") or ("global mobility" and "human resource management") or ("global mobility" and "HRM") or ("global mobility" and "human resource management") or "global HRM" or "global human resource management" or ("talent management" and "globalization") or ("talent management" and "globalisation") or ("international business travellers" and "HRM") or ("international business travellers" and "human resource management") or ("migration" and "HRM") or ("migration" and "human resource management"))
\end{abstract}

Fuente: Elaboración propia

En segundo lugar, se depuran las palabras mal escritas o duplicadas. En tercer lugar, la normalización y la creación de la etapa de red donde se seleccionó la elección de coocurrencia para obtener las redes, y el índice de equivalencia para estandarizar la red. En cuarto lugar, en la etapa de construcción del mapa, el algoritmo de centros simples permite identificar temas o clústeres de temas. En quinto lugar, en la etapa de análisis y visualización, se ofrecen varios diagramas estratégicos, clúster y mapas de evolución. En esta etapa, los temas resultantes se representan utilizando dos herramientas gráficas diferentes: diagramas estratégicos y clústeres de redes temáticas (Cobo et al., 2011a). Se utilizan dos medidas para caracterizar cada uno de los temas (Callon et al., 1991), son la centralidad y la densidad. La centralidad señala las interacciones entre redes y puede considerarse como un indicador de la importancia del tema en el desarrollo del campo. Por otro lado, la densidad representa la fuerza interna de la red y se interpreta como el grado de desarrollo del tema. Tomando como eje horizontal a la centralidad y como eje vertical a la densidad, se obtiene un diagrama estratégico bidimensional con cuatro grupos. Los grupos representados en el mapa estratégico son "temas motores" (temas relevantes y bien desarrollados), "temas especializados o periféricos" (temas irrelevantes pero bien desarrollados), "temas emergentes o en desaparición" (débilmente desarrollados, al observar su evolución en períodos anteriores se puede ver, si estos temas están surgiendo o desapareciendo) y "temas básicos o transversales" (relevantes pero no desarrollados) (Cobo et al., 2012) (ver figura 2). 


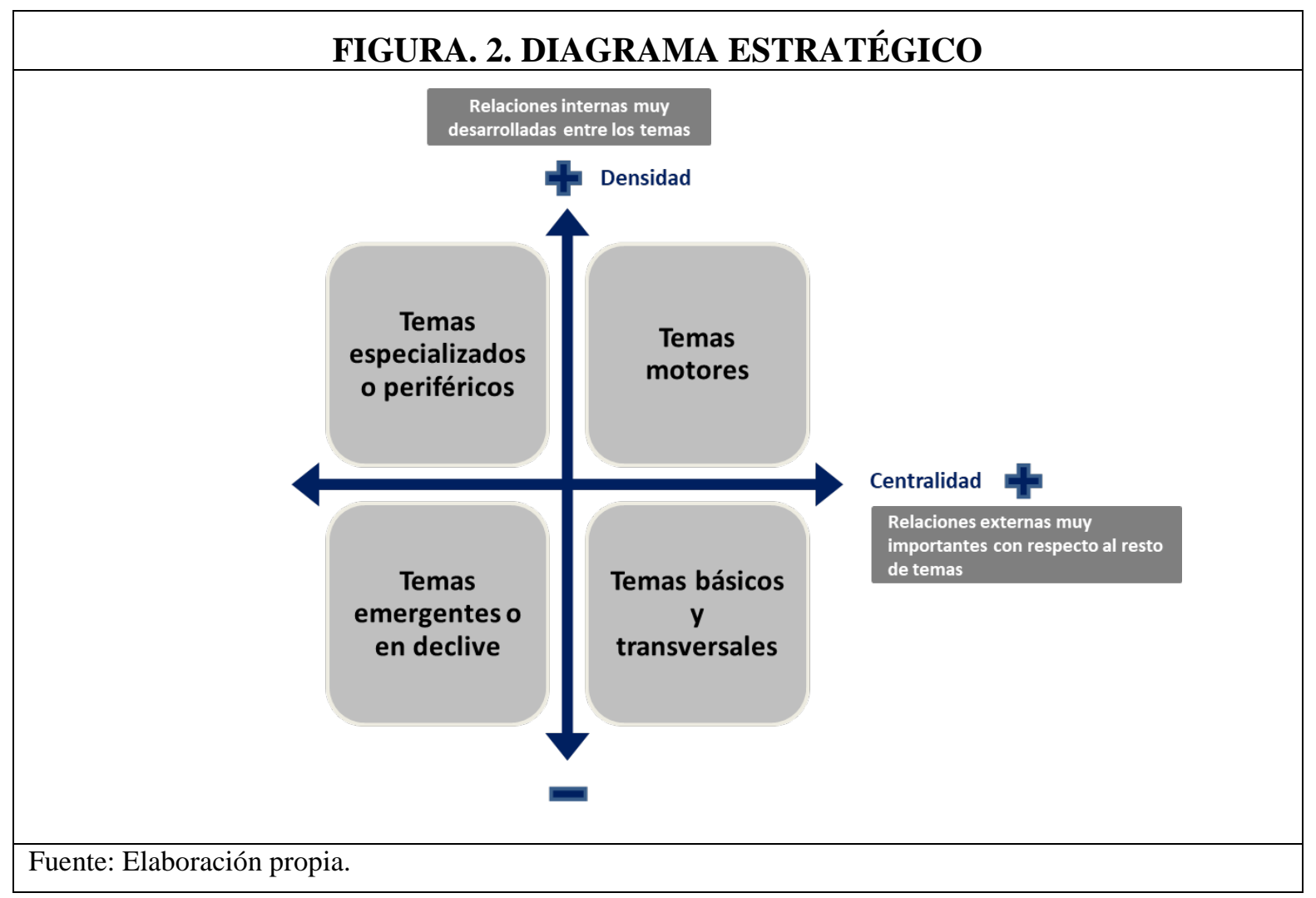

Además, otra herramienta gráfica ofrecida por SciMAT es el mapa de evolución que permite un estudio longitudinal de los temas en los diferentes períodos. La última etapa en el análisis del mapeo científico con la herramienta SciMAT, es un análisis del rendimiento del campo de estudio que ofrece varias medidas bibliométricas como citas, índice h o número de documentos.

\section{RESULTADOS DEL MAPEO CIENTÍFICO DEL CAMPO DE LA GESTIÓN INTERNACIONAL DE LOS RECURSOS HUMANOS.}

La Gestión Internacional de los Recursos Humanos recibe cada vez más de atención como demuestra el hecho que desde 1974 hasta el primer trimestre de 2020, 1.707 artículos tratan sobre este tópico, siendo los artículos y los autores más citados los mostrados en las tablas 2 y 3 respectivamente y las revistas más prolíficas las indicadas en la tabla 4. 


\begin{tabular}{|c|c|c|c|}
\hline \multicolumn{4}{|c|}{$\begin{array}{l}\text { TABLA 2. CLASIFICACIÓN DE LOS } 25 \text { ARTÍCULOS MÁS CITADOS EN LA } \\
\text { WOS EN EL CAMPO DE LA GESTIÓN INTERNACIONAL DE LOS RECURSOS } \\
\text { HUMANOS }\end{array}$} \\
\hline Puesto & Título & Autor/es & $\begin{array}{l}\mathrm{N}^{\mathrm{a}} \mathrm{de} \\
\text { Citas }\end{array}$ \\
\hline 1 & $\begin{array}{l}\text { A Quarter Century Of Culture's Consequences: A Review Of Empirical } \\
\text { Research Incorporating Hofstede's Cultural Values Framework }\end{array}$ & $\begin{array}{l}\text { (Kirkman, Lowe, \& } \\
\text { Gibson, 2006) }\end{array}$ & 898 \\
\hline 2 & $\begin{array}{l}\text { Input-Based And Time-Based Models Of International Adjustment: Meta- } \\
\text { Analytic Evidence And Theoretical Extensions }\end{array}$ & $\begin{array}{l}\text { (Bhaskar-Shrinivas, } \\
\text { Harrison, Shaffer, \& } \\
\text { Luk, 2005) }\end{array}$ & 511 \\
\hline 3 & $\begin{array}{l}\text { Influences On Human-Resource Management-Practices In Multinational- } \\
\text { Corporations }\end{array}$ & $\begin{array}{l}\text { (Rosenzweig \& } \\
\text { Nohria, 1994) }\end{array}$ & 436 \\
\hline 4 & An Institutional Approach To Cross-National Distance & $\begin{array}{l}\text { (Berry, Guillén, \& } \\
\text { Zhou, 2010) }\end{array}$ & 432 \\
\hline 5 & Work Role Transitions - A Study Of American Expatriate Managers in Japan & (Black, 1988) & 430 \\
\hline 6 & $\begin{array}{l}\text { Conceptualizing And Measuring Cultures And Their Consequences: A } \\
\text { Comparative Review Of GLOBE's and Hofstede's Approaches }\end{array}$ & $\begin{array}{l}\text { (Javidan, House, } \\
\text { Dorfman, Hanges, } \\
\text { \& De Luque, 2006) }\end{array}$ & 332 \\
\hline 7 & $\begin{array}{l}\text { Advances In Career Theory And Research: A Critical Review and Agenda } \\
\text { For Future Exploration }\end{array}$ & $\begin{array}{l}\text { (Sullivan \& Baruch, } \\
\text { 2009) }\end{array}$ & 325 \\
\hline 8 & $\begin{array}{l}\text { Changing Patterns Of Global Staffing In The Multinational Enterprise: } \\
\text { Challenges To The Conventional Expatriate Assignment And Emerging } \\
\text { Alternatives }\end{array}$ & $\begin{array}{l}\text { (Collings, Scullion, } \\
\text { \& Morley, 2007) }\end{array}$ & 321 \\
\hline 9 & $\begin{array}{l}\text { Dimensions, Determinants, and Differences in the Expatriate Adjustment } \\
\text { Process }\end{array}$ & $\begin{array}{l}\text { (Shaffer, Harrison, } \\
\text { \& Gilley, 1999) }\end{array}$ & 316 \\
\hline 10 & $\begin{array}{l}\text { Toward an Integrative Model of Strategic International Human Resource } \\
\text { Management }\end{array}$ & $\begin{array}{l}\text { (Taylor, Beechler, } \\
\text { \& Napier, 1996) }\end{array}$ & 314 \\
\hline 11 & Expatriate Managers and the Psychological Contract & $\begin{array}{l}\text { (Guzzo, Noonan, \& } \\
\text { Elron, 1994) }\end{array}$ & 312 \\
\hline 12 & $\begin{array}{l}\text { Managing Knowledge Transfer In MNCs: The Impact of Headquarters } \\
\text { Control Mechanisms }\end{array}$ & $\begin{array}{l}\text { (Björkman, Barner- } \\
\text { Rasmussen, \& Li, } \\
\text { 2004) }\end{array}$ & 305 \\
\hline 13 & $\begin{array}{l}\text { Global Talent Management: Literature Review, Integrative Framework, And } \\
\text { Suggestions For Further Research }\end{array}$ & $\begin{array}{l}\text { (Tarique \& Schuler, } \\
\text { 2010) }\end{array}$ & 287 \\
\hline 14 & $\begin{array}{l}\text { Cross-Cultural Competence In International Business: Toward A Definition } \\
\text { And A Model }\end{array}$ & $\begin{array}{l}\text { (Johnson, } \\
\text { Lenartowicz, \& } \\
\text { Apud, 2006) }\end{array}$ & 279 \\
\hline 15 & $\begin{array}{l}\text { You Can Take It With You: Individual Differences and Expatriate } \\
\text { Effectiveness }\end{array}$ & $\begin{array}{l}\text { (Shaffer, Gregersen, } \\
\text { Ferzandi, Harrison, } \\
\text { \& Black, 2006) }\end{array}$ & 276 \\
\hline 16 & $\begin{array}{l}\text { The Big Five Personality Characteristics as Predictors of Expatriate's Desire } \\
\text { To Terminate the Assignment and Supervisor-Rated Performance }\end{array}$ & (Caligiuri, 2000) & 265 \\
\hline 17 & $\begin{array}{l}\text { Embedded Firms, Embedded Knowledge: Problems Of Collaboration And } \\
\text { Knowledge Transfer in Global Cooperative Ventures }\end{array}$ & (Lam, 1997) & 256 \\
\hline 18 & $\begin{array}{l}\text { Foreign Experience of Top Management Teams And International } \\
\text { Diversification Strategies Of Us Multinational Corporations }\end{array}$ & (Sambharya, 1996) & 251 \\
\hline 19 & American Expatriates Abroad: From Neophytes To Cosmopolitans & (Tung, 1998) & 251 \\
\hline 20 & $\begin{array}{l}\text { Antecedents To Cross-Cultural Adjustment For Expatriates In Pacific Rim } \\
\text { Assignments }\end{array}$ & $\begin{array}{l}\text { (Black \& } \\
\text { Gregersen, 1991) }\end{array}$ & 250 \\
\hline 21 & $\begin{array}{l}\text { Toward The Boundaryless Career: A Closer Look At The Expatriate Career } \\
\text { Concept And The Perceived Implications Of An International Assignment }\end{array}$ & $\begin{array}{l}\text { (Stahl, Miller, \& } \\
\text { Tung, 2002) }\end{array}$ & 239 \\
\hline 22 & The Role Of The Corporate HR Function In Global Talent Management & $\begin{array}{l}\text { (Farndale, Scullion, } \\
\text { \& Sparrow, 2010) }\end{array}$ & 234 \\
\hline 23 & $\begin{array}{l}\text { Of Bears, Bumble-Bees, And Spiders: The Role Of Expatriates In } \\
\text { Controlling Foreign Subsidiaries }\end{array}$ & (Harzing, 2001) & 227 \\
\hline 24 & $\begin{array}{l}\text { Expatriates' Psychological Withdrawal From International Assignments: } \\
\text { Work, Nonwork, And Family Influences }\end{array}$ & $\begin{array}{l}\text { (Shaffer \& } \\
\text { Harrison, 1998) }\end{array}$ & 219 \\
\hline 25 & $\begin{array}{l}\text { Recent Developments In International Management Research: A Review Of } \\
20 \text { Top Management Journals }\end{array}$ & (Werner, 2002) & 214 \\
\hline
\end{tabular}




\section{TABLA 3. CLASIFICACIÓN DE LOS 30 AUTORES CON MAYOR PRODUCTIVIDAD EN LA WOS EN EL CAMPO DE LA GESTIÓN INTERNACIONAL DE LOS RECURSOS HUMANOS}

\begin{tabular}{|c|c|c|}
\hline Puesto & Autores & Documentos \\
\hline 1 & Brewster, Chris & 25 \\
\hline 2 & Dickmann, Michael & 22 \\
\hline 3 & Lauring, Jakob & 20 \\
\hline 4 & Selmer, Jan & 19 \\
\hline 5 & Sparrow, Paul & 19 \\
\hline 6 & Schuler, Randall S. & 16 \\
\hline 7 & Collings, David G. & 16 \\
\hline 8 & Scullion, Hugh & 16 \\
\hline 9 & Farndale, Elaine & 15 \\
\hline 10 & McNulty, Yvonne & 13 \\
\hline 11 & Cerdin, Jean-Luc & 12 \\
\hline 12 & Tarique, Ibraiz & 12 \\
\hline 13 & Shen, Jie & 11 \\
\hline 14 & Suutari, Vesa & 11 \\
\hline 15 & Jackson, Susan E. & 11 \\
\hline 16 & Haslberger, Arno & 11 \\
\hline 17 & Budhwar, Pawan & 10 \\
\hline 18 & Baruch, Yehuda & 10 \\
\hline 20 & Shaffer, Margaret A. & 10 \\
\hline 21 & Hutchings, Kate & 10 \\
\hline 22 & Jackson, Terence & 10 \\
\hline 23 & De Cieri, Helen & 10 \\
\hline 24 & Al Ariss, Akram & 10 \\
\hline 25 & Shortland, Susan & 9 \\
\hline 26 & Bonache, Jaime & 9 \\
\hline 27 & Stahl, Guenter K. & 9 \\
\hline 28 & Caligiuri, Paula & 9 \\
\hline 29 & Harzing, Anne-Wil & 9 \\
\hline 30 & Tung, Rosalie L. & 9 \\
\hline
\end{tabular}

Fuente: Elaboración propia. 


\begin{tabular}{|c|c|c|}
\hline \multicolumn{3}{|c|}{$\begin{array}{l}\text { TABLA 4. CLASIFICACIÓN DE LAS REVISTAS MÁS PRODUCTIVAS EN EL } \\
\text { CAMPO DE LA GESTIÓN INTERNACIONAL DE LOS RECURSOS HUMANOS }\end{array}$} \\
\hline & Revista & $\begin{array}{l}\text { Número de } \\
\text { Documentos }\end{array}$ \\
\hline 1 & International Journal Of Human Resource Management & 318 \\
\hline 2 & Journal Of Global Mobility-The Home Of Expatriate Management Research & 77 \\
\hline 3 & Journal Of World Business & 72 \\
\hline 4 & International Journal Of Cross Cultural Management & 67 \\
\hline 5 & Human Resource Management & 63 \\
\hline 6 & Journal Of International Business Studies & 51 \\
\hline 7 & Personnel Review & 41 \\
\hline 8 & Thunderbird International Business Review & 31 \\
\hline 9 & European Journal Of International Management & 26 \\
\hline 10 & Journal Of Management Development & 26 \\
\hline 11 & Human Resource Management Review & 25 \\
\hline 12 & Cross Cultural Management-An International Journal & 24 \\
\hline 13 & Journal Of Business Ethics & 23 \\
\hline 14 & Cross Cultural \& Strategic Management & 20 \\
\hline 15 & Human Relations & 19 \\
\hline 16 & International Business Review & 19 \\
\hline 17 & Management International Review & 19 \\
\hline 18 & Career Development International & 17 \\
\hline 19 & International Journal Of Manpower & 16 \\
\hline 20 & Handbook Of Research In International Human Resource Management & 16 \\
\hline 21 & Asia Pacific Journal Of Human Resources & 15 \\
\hline 22 & Journal Of Management & 15 \\
\hline 23 & Journal Of International Management & 15 \\
\hline 24 & Employee Relations & 14 \\
\hline 25 & Journal Of Managerial Psychology & 13 \\
\hline 26 & Human Resource Development International & 12 \\
\hline 27 & International Journal Of Organizational Analysis & 12 \\
\hline 28 & Journal Of Business Research & 12 \\
\hline 29 & Journal Of Organizational Change Management & 12 \\
\hline 30 & International Marketing Review & 11 \\
\hline 31 & European Management Journal & 10 \\
\hline 32 & Management Decision & 10 \\
\hline 33 & Academy Of Management Learning \& Education & 10 \\
\hline 34 & Organization Studies & 10 \\
\hline 35 & Asia Pacific Business Review & 9 \\
\hline
\end{tabular}

En el período comprendido entre 1974 y 1993, aparecen 35 documentos relacionados con la temática; entre 1994 y 2003, 164 documentos; de 2004 a 2009, 350 manuscritos; de 2010 a 2014, 549 manuscritos; y de 2015 hasta marzo de 2020, se obtienen 609 (ver figura. 3), por 
tanto, en los últimos 10 años el número de publicaciones sobre la Gestión Internacional de los Recursos Humanos se ha casi duplicado.

\section{FIGURA 3. PUBLICACIONES INTERNACIONALES DE LA WOS SOBRE LA GESTIÓN INTERNACIONAL DE LOS RECURSOS HUMANOS ENTRE 1974 Y 2020}

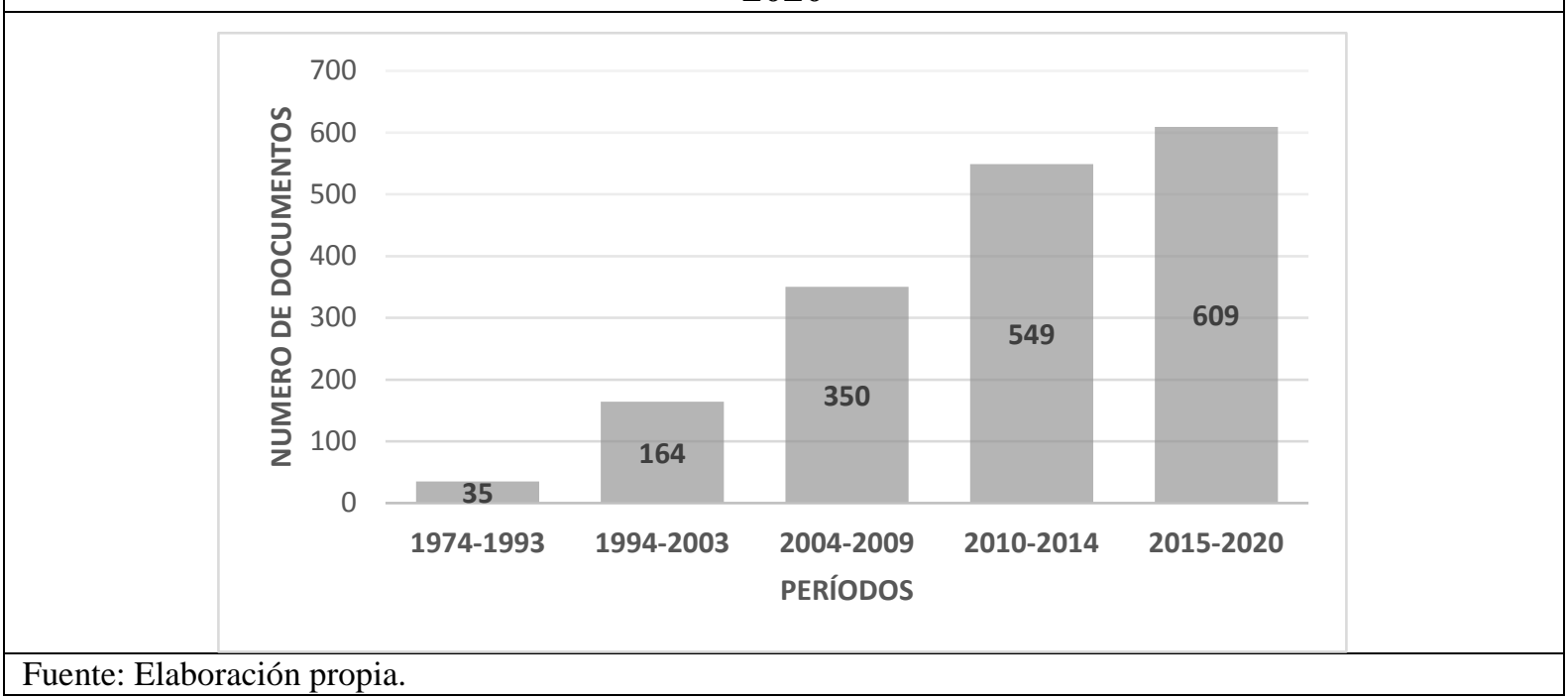

\subsection{Evolución de los temas sobre la Gestión Internacional de los RRHH}

La figura 4 presenta la evolución del campo de estudio de la Gestión Internacional de los Recursos Humanos en los siguientes períodos: 1974-1993, 1994-2003, 2004-2009, 2010-2014, 2015-2020.

FIGURA 4. MAPA DE EVOLUCIÓN DEL CAMPO DE ESTUDIO DE LA GESTIÓN INTERNACIONAL DE LOS RECURSOS HUMANOS EN LOS PERÍODOS 1974-1993, 1994-2003, 2004-2009, 2010-2014, 2015-2020

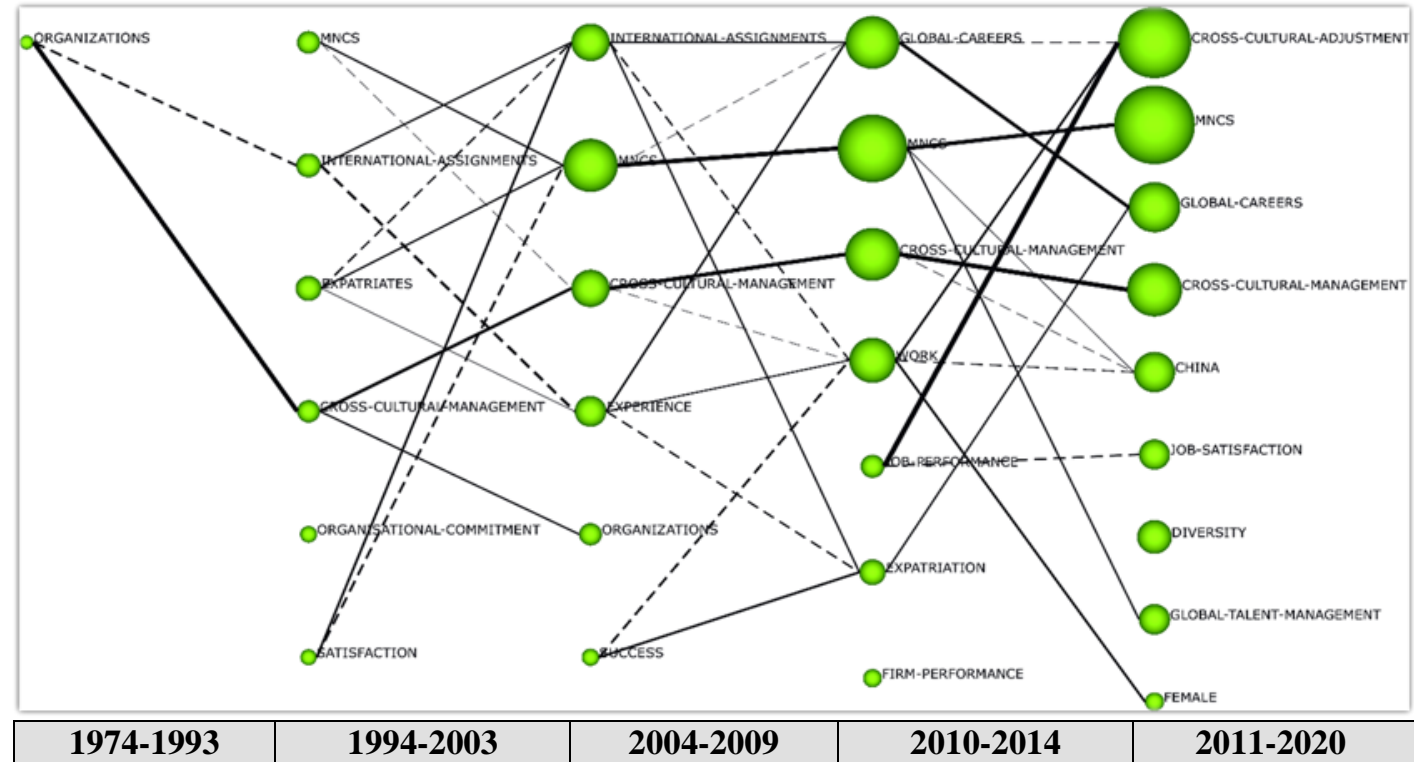

Fuente: Elaboración propia. 
Las palabras claves van variando en los diferentes subperíodos (1974-1993, 1994-2003, 2004-2009, 2010-2014, 2015-2020) (figura 4). La terminología de la temática sobre la Gestión Internacional de los Recursos Humanos va evolucionando utilizando diferentes palabras claves para describir el contenido de los distintos documentos. Así, en el último período 2015-2020, nuevas palabras claves surgen como clústeres (mujeres, gestión del talento global, diversidad, satisfacción) y, otro tema como expatriación se incorpora al tema relacionado, carreras globales. En el período 2010-14, el tema asignaciones internacionales se incorpora a los temas de carreras globales y expatriación. Por otro lado, temas como la gestión intercultural o las multinacionales han permanecido inalterados durante cuatro períodos (1994-2003, 2004-2009, 2010-2014, 2015-2020), asignaciones internacionales se ha mantenido durante dos períodos consecutivos (1994-2003, 2004-2009) y carreras globales ha tomado relevancia en los dos últimos períodos (2010-2014, 2015-2020).

\subsection{Tópicos de la Gestión Internacional de los Recursos Humanos por períodos}

Temas de la Gestión Internacional de los Recursos Humanos en el período 1974-1993

En el período comprendido entre 1974 y 1993 solo se observa un clúster que relaciona la importancia en las organizaciones de aspectos relacionados con el trabajo y la cultura (ver figura 5). El trabajo de Hofstede (1980) supuso una importantísima aportación al campo de la gestión intercultural y su aplicación a la Gestión Internacional de los Recursos Humanos (Hofstede, 1993; Schuler et al., 1993). Inicialmente, Geert Hofstede (1980) propuso cuatro dimensiones en las que se podían entender las diferencias entre las culturas nacionales: individualismo, distancia de poder, evitación de la incertidumbre y masculinidad. Posteriormente, se incluyeron dos nuevas dimensiones relativas a la orientación temporal y al nivel indulgencia. La aplicación del modelo de Hofstede a la Gestión Internacional de los Recursos Humanos, le dio un impulso muy importante a esta disciplina (Banai y Reisel, 1993; Black, 1992; Brewster, 1991; Dowling y Schuler, 1990; Schuler et al., 1993; Schuler et al., 1991) y marcó un antes y un después en la misma. Por tanto, las aportaciones de este primer período, 1974-2003 supondrán un importante cimiento para el desarrollo del campo científico de la Gestión Internacional de los Recursos Humanos.

FIGURA 5. RED DE CLÚSTER DE LA GESTIÓN INTERNACIONAL DE LOS RECURSOS HUMANOS (ORGANIZACIÓN) PARA EL PERÍODO 1974-1993

Fuente: Elaboración propia. 
Temas de la Gestión Internacional de los Recursos Humanos en el período 1994-2003

En el período 1994-2003, los temas motores (bien desarrollado e importantes para el campo científico) de la Gestión Internacional de los Recursos Humanos son expatriados y asignaciones internacionales, los temas básicos (importantes, pero no suficientemente desarrollados) son el compromiso organizativo y la gestión intercultural. Satisfacción sería un tema emergente (no desarrollado y marginal para el resto de temas de su disciplina) y la problemática relativa a las multinacionales sería un tema especializado (desarrollado, pero no central para el resto de temas de su campo) (figura 6). El ajuste de los expatriados en su asignación internacional fue objeto de estudio en este período, confirmando la multidimensionalidad de ese ajuste (Shaffer et al., 1999), las características de los expatriados que determinan su deseo de finalizar su asignación internacional (Caligiuri, 2000) o las prácticas que pueden emplear las organizaciones con sus directivos expatriados y su impacto en los resultados para la retención de los mismos (como, por ejemplo, el compromiso organizativo o la intención de abandonar la asignación) (Guzzo et al., 1994). También, la gestión intercultural y su aplicación al campo de la Gestión Internacional de los Recursos Humanos, van adquiriendo relevancia en este período, en este sentido, Budhwar y Sparrow (2002) indican que las estrategias de gestión de los Recursos Humanos varían mucho cuando se considera el contexto internacional, la implementación de estas estrategias de Recursos Humanos a nivel internacional pueden tener resultados muy diferentes y, por tanto, se debe tener en cuenta a la hora de diseñar estrategias internacionales de Recursos Humanos.

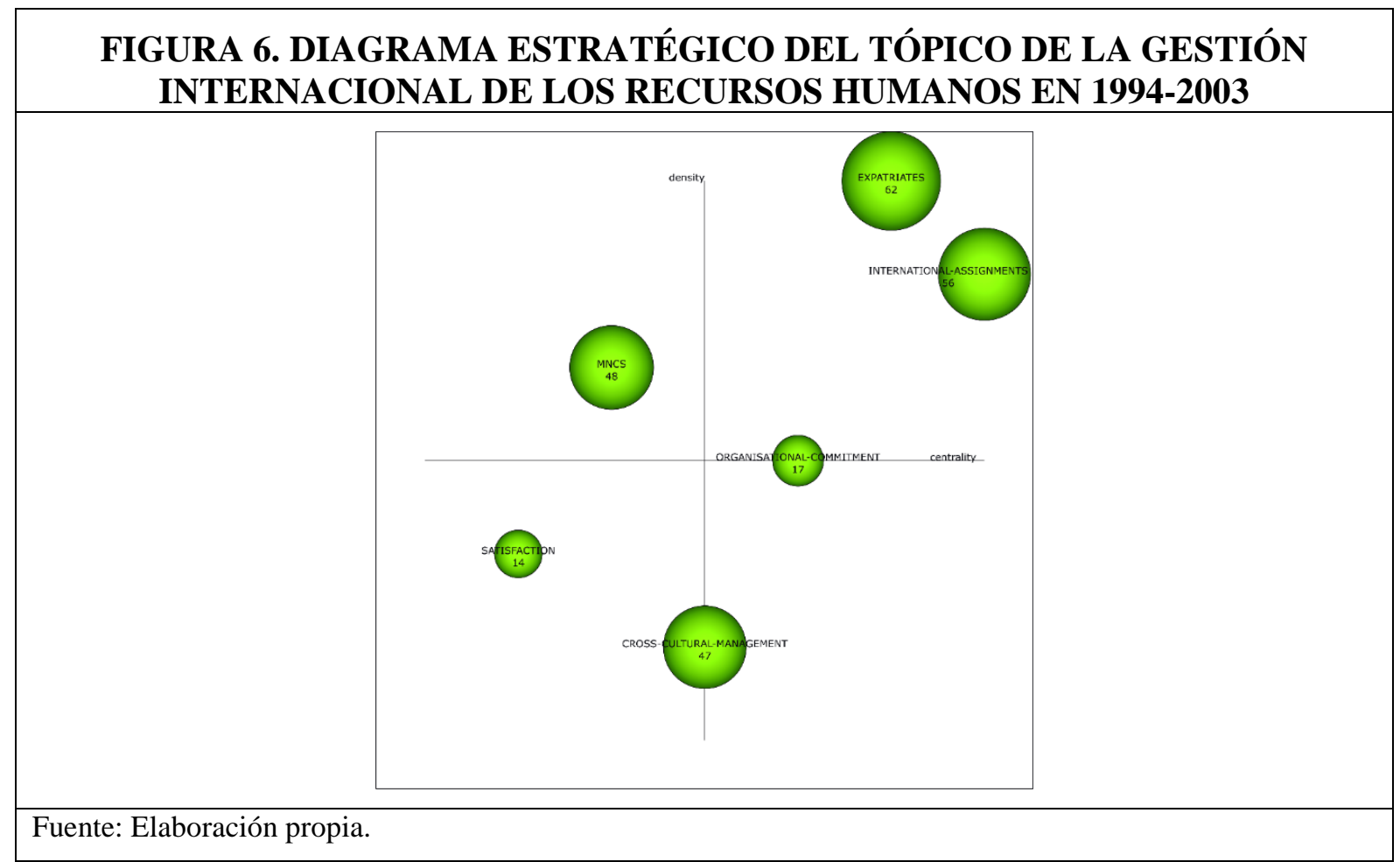

Temas de la Gestión Internacional de los Recursos Humanos en el período 2004-2009

Entre 2004 y 2009, los temas motores son multinacionales, asignaciones internacionales y experiencia con 207, 126 y 91 documentos respectivamente. La gestión intercultural, con 125 documentos en este período, sería un tema básico o transversal para la disciplina. Los aspectos 
relacionados con el éxito del proceso de expatriación son emergentes y los aspectos meramente organizativos van perdiendo relevancia en la Gestión Internacional de los Recursos Humanos (figura 7). En efecto el tema de las asignaciones internacionales se ha consolidado en el campo de la Gestión Internacional de los Recursos Humanos (Collings et al., 2007). Las multinacionales están inmersas en una creciente complejidad en el entorno global especialmente en los países emergentes donde las estrategias de Recursos Humanos impactan drásticamente entre los empleados locales (Collings et al., 2007; Tung, 1998), esto lleva a muchas organizaciones a plantearse la incorporación de nuevas formas de trabajo internacional, además de la expatriación (Morley y Heraty, 2004). En este período, los estudios también han avanzado en el análisis de la influencia de las experiencias de expatriados y repatriados en el desarrollo de la carrera profesional y en la retención del expatriado (Kraimer et al., 2009), estos autores diseñaron un modelo para analizar esta relación y también encontraron que las tareas de desarrollo de expatriados estaban positivamente relacionadas con el avance profesional.

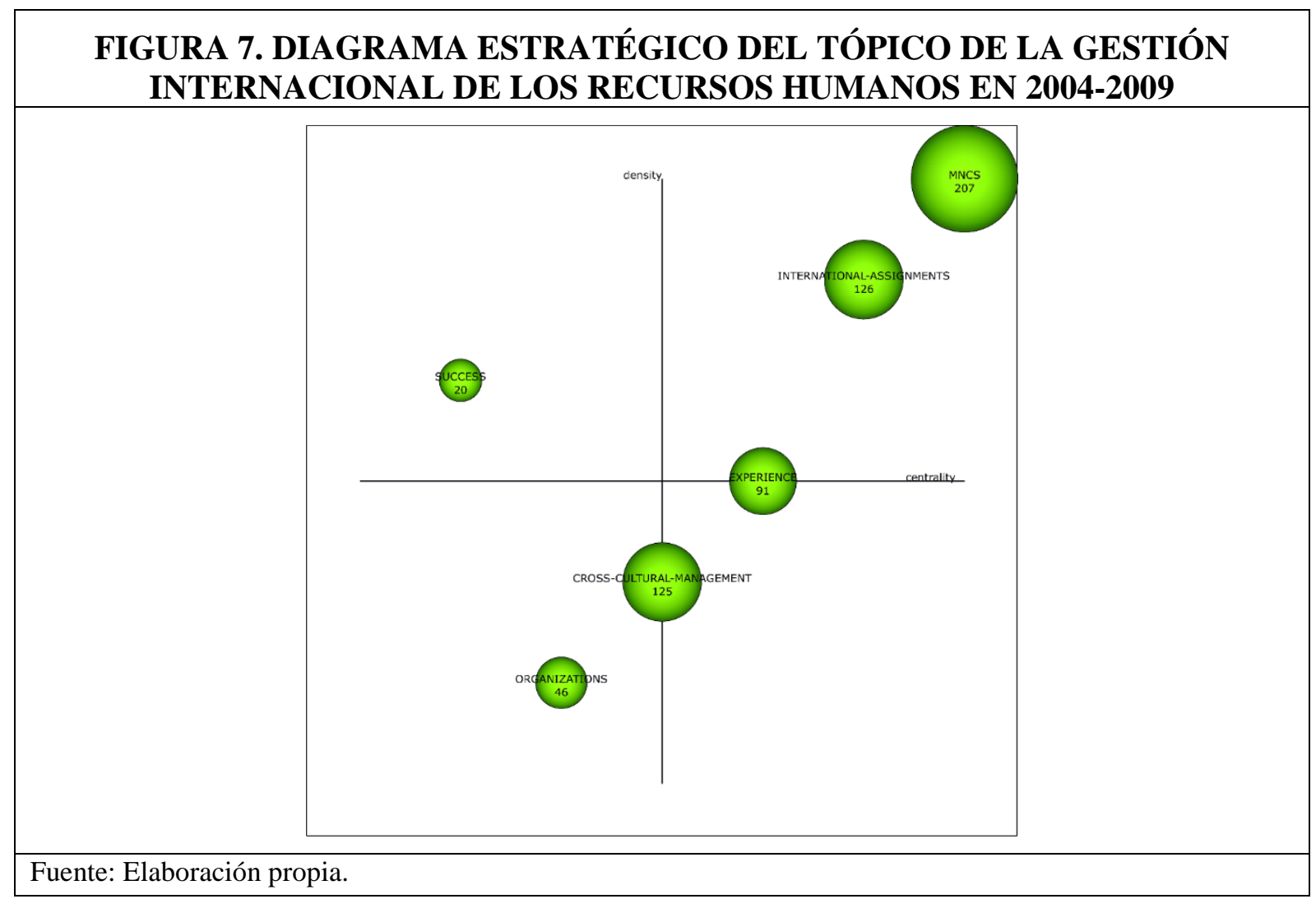

Temas de la Gestión Internacional de los Recursos Humanos en el período 2010-2014

En el período 2010-2014, los temas relativos a carreras globales y a multinacionales constituyen temas motores. La gestión intercultural y el trabajo constituyen temas básicos o transversales en el campo de la Gestión Internacional de los Recursos Humanos. La expatriación emerge como un clúster independiente, hasta ahora (en períodos previos) estaba incluido en el tema de las asignaciones internacionales. Por último, temas relacionados al rendimiento de la empresa o al desempeño laboral son temas más especializados dentro de la Gestión Internacional de los Recursos Humanos (figura 8). 


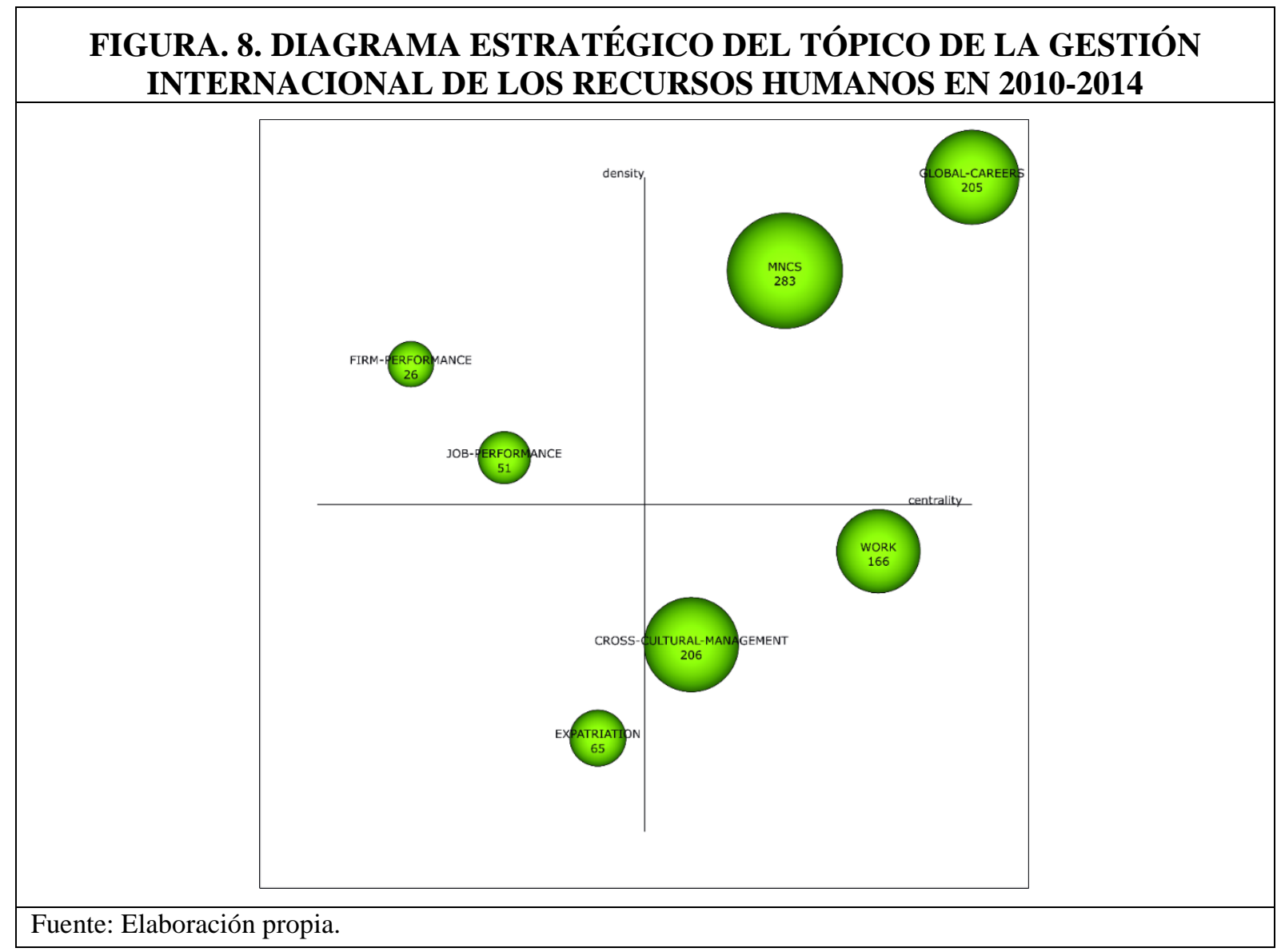

Este período 2010-2014 cuenta con un volumen significativo de documentos (ver figura. 3) con 549 manuscritos. En la tabla 5, se presenta un análisis del rendimiento de este período.

\begin{tabular}{|c|c|c|c|}
\hline \multicolumn{4}{|c|}{$\begin{array}{l}\text { TABLA 5. ANÁLISIS DEL RENDIMIENTO DE LOS TEMAS DE GESTIÓN } \\
\text { INTERNACIONAL DE LOS RECURSOS HUMANOS EN EL PERÍODO 2010-2014 }\end{array}$} \\
\hline Tema & Documentos & Citas & h-Index \\
\hline Carreras globales & 205 & 5.166 & 39 \\
\hline Multinacionales & 283 & 6.360 & 39 \\
\hline Gestión Intercultural & 206 & 4.394 & 30 \\
\hline Trabajo & 166 & 3.626 & 33 \\
\hline Desempeño laboral & 51 & 1.149 & 21 \\
\hline Expatriación & 65 & 1.599 & 23 \\
\hline $\begin{array}{l}\text { Rendimiento de la } \\
\text { empresa }\end{array}$ & 26 & 536 & 12 \\
\hline
\end{tabular}

El tema de las carreras globales irrumpe como un clúster independiente en este período 2010-2014 (figura 9), previamente estaba incluido dentro de international assignments (ver figura 4). 


\section{FIGURA 9. RED DE CLÚSTER DE LA GESTIÓN INTERNACIONAL DE LOS RECURSOS HUMANOS (CARRERAS GLOBALES) PARA EL PERÍODO 2010- 2014}

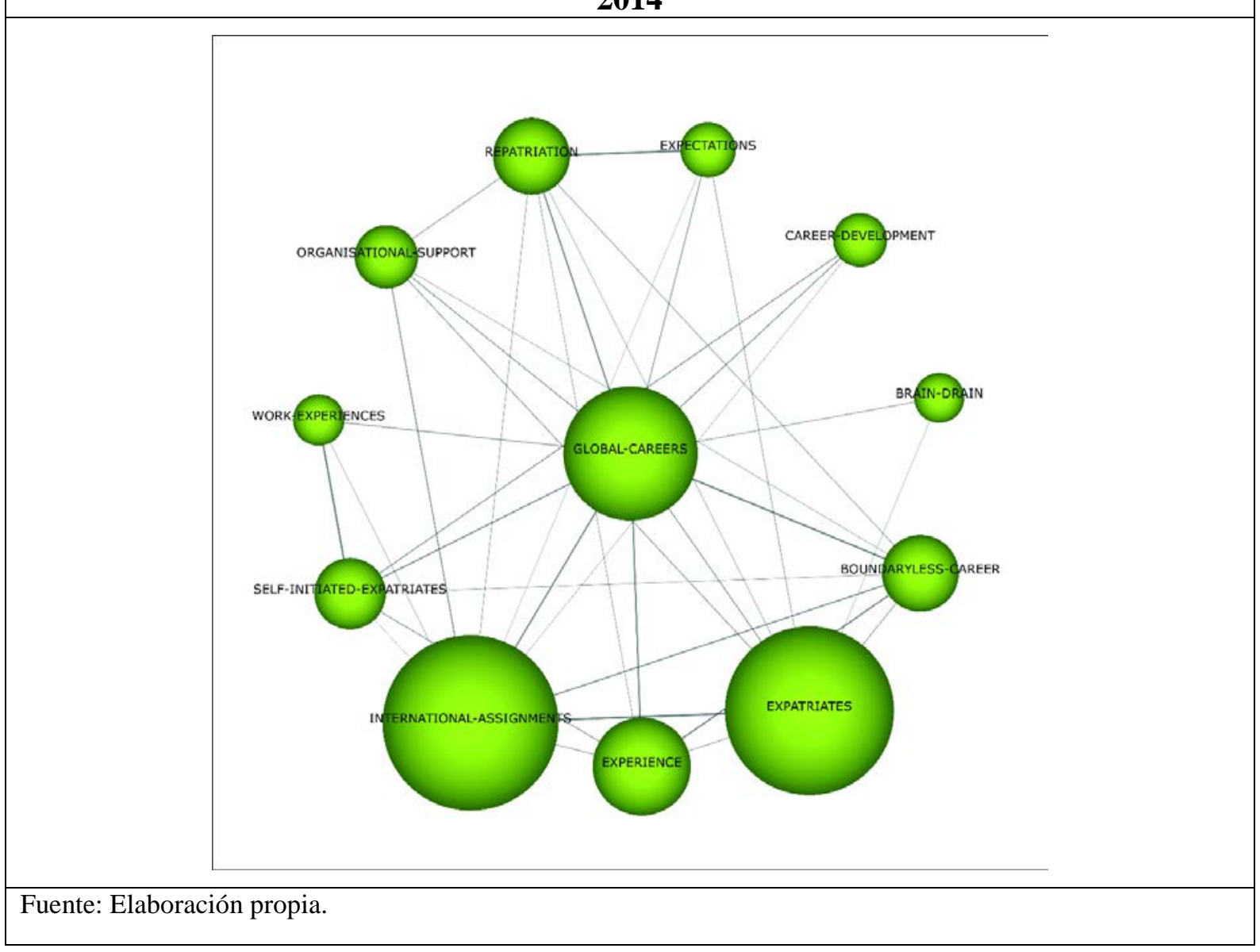

Temas relativos a los expatriados autoiniciados, la repatriación, las experiencias o el desarrollo de la carrera profesional internacional forman parte del clúster del tema de carreras globales. Ante la complejidad de gestionar los procesos de expatriación y repatriación, muchas organizaciones tratan de buscar formas alternativas de trabajo internacional como, por ejemplo, las fórmulas de expatriación auto iniciadas, los flexpatriados, las asignaciones en períodos cortos o los viajeros internacionales de negocios (Shaffer et al., 2012). Muchos autores consideran que estás formas alternativas de trabajo internacional se refieren a las carreras globales (Cerdin y Bird, 2008), pero de acuerdo con Shaffer et al. (2012) constituyen más bien experiencias de trabajo globales, más que carreras globales. En este período, también, la problemática relacionada con las multinacionales sigue siendo relevante. En efecto, el impacto de la distancia intercultural es crucial a la hora de adoptar la decisión de si entrar o no en un país (Berry et al., 2010), la comprensión de cómo abordan las multinacionales las presiones de la globalización en lo que respecta a la gestión de los Recursos Humanos sigue ocupando las investigaciones (Farndale, Paauwe, et al., 2010). En este sentido, Farndale et al. (2010), desarrollaron una modelo contextual que recoge configuraciones corporativas de las funciones de la gestión de Recursos Humanos en las multinacionales, aportando incluso nuevas ideas para diseñar prácticas de Gestión Internacional de los Recursos Humanos. 
Temas de la Gestión Internacional de los Recursos Humanos en el período 2015-2020

En este último período, 2015-2020, el ajuste intercultural, las multinacionales, las carreras globales y la gestión intercultural representan temas motores (importantes para su disciplina y desarrollados). La problemática de la Gestión Internacional de los Recursos Humanos en China constituye un tema transversal (central para su disciplina, pero que aún puede desarrollarse más). La gestión global del talento, las mujeres en el contexto internacional o la diversidad son temas emergentes de esta disciplina. Por último, la satisfacción laboral es un tema especializado (desarrollado, pero no central para esta disciplina) (figura 10).

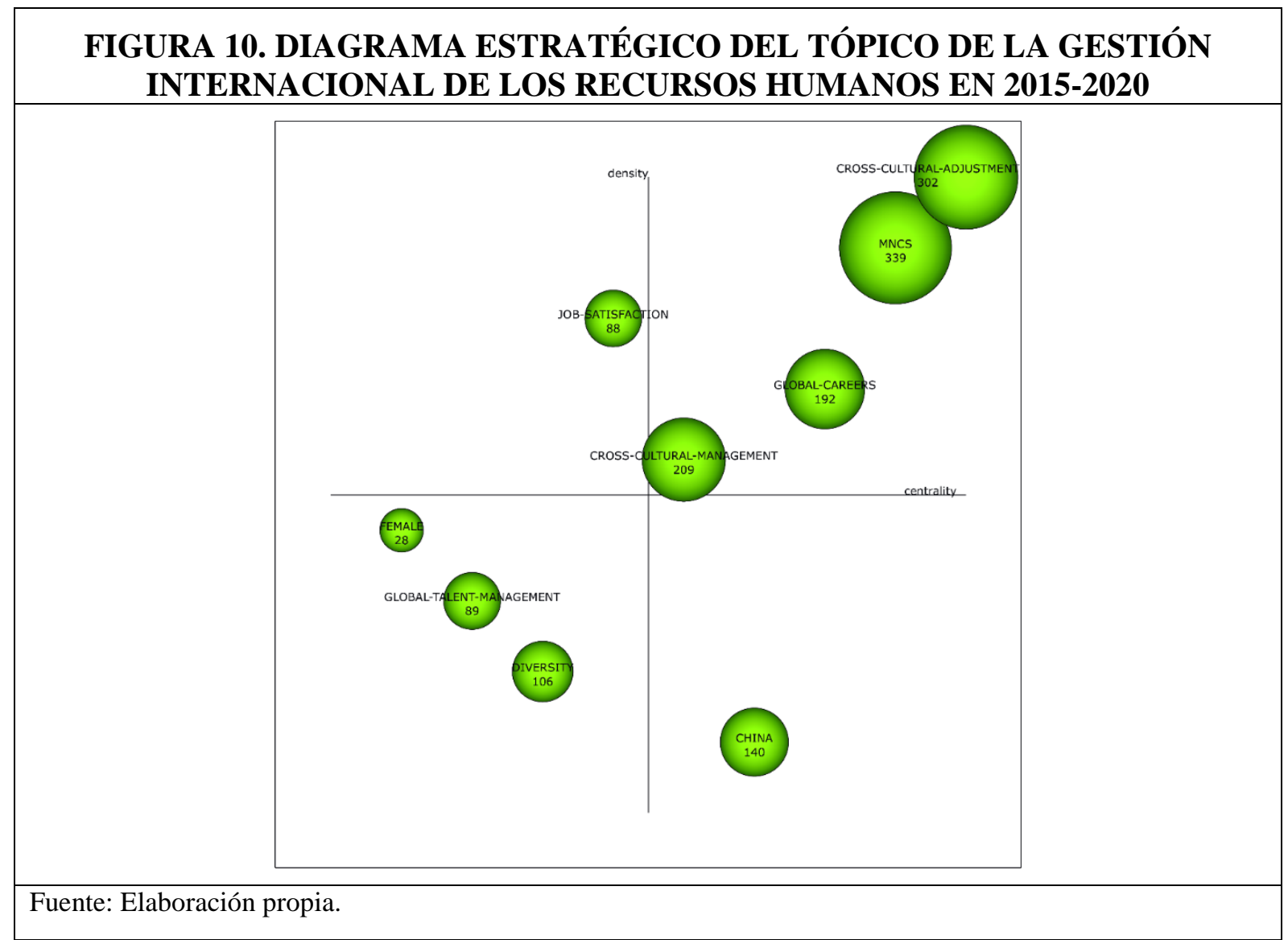

Al igual que el período anterior, en esta etapa, 2015-2020, la productividad en el tópico de la Gestión Internacional de los Recursos Humanos es elevada, con 609 documentos hasta febrero de 2020 (figura 3). En la tabla 6, se presenta un análisis del rendimiento de la disciplina de la Gestión Internacional de los Recursos Humanos entre 2015 y 2020. 


\section{TABLA 6. ANÁLISIS DEL RENDIMIENTO DE LOS TEMAS DE LA GESTIÓN INTERNACIONAL DE LOS RECURSOS HUMANOS EN EL PERÍODO 2015-2020}

\begin{tabular}{|l|l|l|l|}
\hline Tema & Documentos & Citas & h-Index \\
\hline Ajuste intercultural & 302 & 1.789 & 20 \\
\hline Multinacionales & 339 & 2.013 & 22 \\
\hline Carreras globales & 192 & 1.326 & 18 \\
\hline Gestión Intercultural & 209 & 1.410 & 17 \\
\hline China & 140 & 752 & 15 \\
\hline Satisfacción laboral & 88 & 344 & 10 \\
\hline Diversidad & 106 & 881 & 15 \\
\hline Gestión Global del Talento & 89 & 666 & 13 \\
\hline Mujeres & 28 & 115 & 6 \\
\hline
\end{tabular}

Fuente: Elaboración propia.

El ajuste intercultural de los expatriados es un tema importante, lo ha sido en períodos anteriores donde este tema estaba incluido en otros clústeres, pero sigue siendo relevante como demuestra el hecho de que ahora el ajuste intercultural es un clúster independiente (figura 10). Hippler et al. (2015) realizan una revisión exhaustiva del ajuste de los expatriados especialmente considerando la dinámica temporal y realizan recomendaciones. Abdul Malek, Budhwar y Reiche (2015) analizan el papel del apoyo percibido de las multinacionales y nacionales del país anfitrión para el ajuste de los expatriados y sus cónyuges, estos autores encontraron diferencias entre el apoyo percibido y el ajuste, indicando que un ajuste mejorado tenía consecuencias positivas para el desempeño de los expatriados. Por otro lado, los aspectos relacionados a la Gestión Internacional de los Recursos Humanos con China, suscitan mucho interés entre los académicos, como demuestra el hecho de que existen 140 documentos y 752 citas referidas a esa temática (tabla 6). Así, Zhong y Chin (2015) estudian aspectos relacionados con la transferencia de conocimiento entre empleados en multinacionales chinas y Ko y Liu (2017) analizan el papel del Guanxi y las redes sociales(uso de los contactos sociales para acceder a recursos) en las prácticas de reclutamiento chinas.

Además, en este período 2015-2020, presentan especial interés los temas emergentes como la gestión del talento global, las mujeres expatriadas y la diversidad. Las compañías internacionales se enfrentan a importantes desafíos para integrar sus estrategias de gestión del talento global (Cascio y Boudreau, 2016) entre una fuerza laboral dispersa y diversa (Morris et al., 2016). Estos autores explican en un modelo cómo se desarrollan los diferentes tipos de capital humano en diferentes niveles (individual, departamental, organizativo) con el fin de lograr una cartera de talento para la multinacional. Dickmann et al. (2018) analizaron el impacto de trabajar en el extranjero sobre el capital profesional de las personas. Por último, entre las razones para la representación insuficiente de las mujeres en las asignaciones internacionales caben citar los estereotipos sobre su capacidad para adaptarse en el extranjero o la falta de confianza desde la sede matriz (Salamin y Davoine, 2015). En efecto, los temas relacionados a las mujeres expatriadas van incrementando su relevancia, así Shen y Jiang (2015) profundizan sobre los factores que influyen el rendimiento de las mujeres expatriadas y Salamin y Davoine (2015) indicaron que las mujeres expatriadas demuestran niveles más altos de interacción y ajuste de trabajo que los hombres en el contexto europeo en consonancia con los resultados de Selmer y Leung (2003). 


\section{DISCUSIÓN, LIMITACIONES Y CONCLUSIONES}

\subsection{Discusión}

Año tras año, el número de empresas con actividad internacional ha crecido, y con ello el número de empleados que las empresas transfieren a sus filiales, la contratación de personal local por empresas multinacionales o las dificultades en la gestión. La literatura académica sigue prestando atención a las experiencias internacionales, ya sea en asignaciones de mayor o menor duración temporal, la gestión del talento global o la complejidad de la Gestión Internacional de los Recursos Humanos. No obstante, la terminología ha evolucionado utilizando diferentes palabras claves para describir el contenido de los distintos estudios a lo largo de los últimos años. Así, los primeros estudios relativos a la Gestión Internacional de los Recursos Humanos se centraban básicamente en la gestión intercultural, y con el paso de los años se incorporaron otras temáticas como las asignaciones internacionales, expatriados o multinacionales.

Resulta especialmente relevante destacar las tendencias del periodo más reciente (20152020) reflejadas por nuestro análisis, pues pueden servir de guía a académicos y directivos para destacar los temas de mayor interés. En ese sentido, surgen nuevas palabras clave con entidad, como mujeres, gestión del talento global, diversidad, satisfacción, mientras que expatriación se incorpora al tema relacionado carreras globales. Los temas motores de estos últimos años (aquellos importantes para la disciplina y que han sido desarrollados) son el ajuste intercultural, gestión intercultural (tema que se mantiene vivo en la disciplina desde los primeros estudios de Hofstede), las multinacionales, y las carreras globales. La aparición de estudios en diferentes contextos queda reflejada en la importancia de los estudios realizados en China, que constituye un tema transversal (central para su disciplina, pero que al que aún le queda desarrollo). Los temas emergentes en la Gestión Internacional de los Recursos Humanos son la gestión global del talento, las mujeres en el contexto internacional y la diversidad, temas de actualidad que tienen mucho desarrollo posible en el campo. Finalmente, la satisfacción laboral aparece como tema especializado (desarrollado, pero no central para esta disciplina).

\subsection{Limitaciones}

La restricción de este análisis a los artículos y revisiones de la WoS en las áreas de Business y Management, aunque ofrece una representación óptima del campo de la Gestión Internacional de los Recursos Humanos, constituye la principal limitación de este estudio. Una segunda limitación de la investigación es que la literatura de trabajo de la Gestión Internacional de los Recursos Humanos es tan prolífica, como demuestra el número de publicaciones a lo largo de estos años, que puede que en los próximos años los trabajos publicados destaquen otras temáticas o contextos. Sin embargo, esta limitación también es una potencial fortaleza futura: a medida que se continúe desarrollando este campo de estudio, será necesario replicar un estudio como el realizado para entender las tendencias en el área de la Gestión Internacional de los Recursos Humanos.

\subsection{Conclusiones}

El presente estudio contribuye al desarrollo de la Gestión Internacional de los Recursos Humanos presentando un análisis bibliométrico y una revisión sistemática que ayuda a aclarar los parámetros analizados desde 1974 hasta la fecha, destacando los temas y tendencias más prometedores para el futuro, como serían temas emergentes tales como la gestión global del talento, las mujeres en el contexto internacional y la diversidad. Este trabajo puede guiar a investigadores, profesionales y administraciones públicas sobre los pasos a seguir en el futuro. 


\section{BIBLIOGRAFÍA}

Abdul Malek, M., Budhwar, P., \& Reiche, B. S. (2015). Sources of support and expatriation: a multiple stakeholder perspective of expatriate adjustment and performance in Malaysia. The International Journal of Human Resource Management, vol. 26, $\mathrm{n}^{\circ}$ 2, pp. 258-276. https://doi.org/10.1080/09585192.2014.937968.

Andersen, N. (2019). Mapping the expatriate literature: a bibliometric review of the field from 1998 to 2017 and identification of current research fronts. International Journal of Human Resource Management. https://doi.org/10.1080/09585192.2019.1661267.

Banai, M., \& Reisel, W. D. (1993). Expatriate Managers' Loyalty to the MNC: Myth or Reality? An Exploratory Study. Journal of International Business Studies, vol. 24, n 2, pp. 233 248. https://doi.org/10.1057/palgrave.jibs.8490231.

Berry, H., Guillén, M. F., \& Zhou, N. (2010). An institutional approach to cross-national distance. Journal of International Business Studies, vol. 41, nº 9, pp. 1460-1480.

Bhaskar-Shrinivas, P., Harrison, D. A., Shaffer, M. A., \& Luk, D. M. (2005). Input-Based and Time-Based Models of International Adjustment: Meta-Analytic Evidence and Theoretical Extensions. The Academy of Management Journal, vol. 48, n 2, pp. 257281. https://doi.org/10.2307/20159655.

Björkman, I., Barner-Rasmussen, W., \& Li, L. (2004). Managing Knowledge Transfer in MNCs: The Impact of Headquarters Control Mechanisms. Journal of International Business Studies, vol. 35, n 5, pp. 443-455. https://doi.org/10.2307/3875204.

Black, J. S. (1988). Work Role Transitions: A Study of American Expatriate Managers in Japan. Journal of International Business Studies, vol. 19, $\mathrm{n}^{\circ}$ 2, pp. 277-294. https://doi.org/10.2307/155026.

Black, J. S. (1992). Socializing American Expatriate Managers Overseas. Group \& Organization Management, vol. 17, $\mathrm{n}^{0}$ 2, pp. 171-192. https://doi.org/10.1177/1059601192172005.

Black, J. S., \& Gregersen, H. B. (1991). Antecedents to Cross-Cultural Adjustment for Expatriates in Pacific Rim Assignments. Human Relations, vol. 44, no 5, pp. 497-515. https://doi.org/10.1177/001872679104400505.

Bornay-Barrachina, M. (2019). International Human Resource Management: How Should Employees Be Managed in an International Context? In Managerial Competencies for Multinational Business, pp. 174-194. IGI Global. https://doi.org/10.4018/978-1-52255781-4.ch009.

Börner, K., Chen, C., \& Boyack, K. W. (2005). Visualizing knowledge domains. Annual Review of Information Science and Technology, vol. 37, $\mathrm{n}^{\circ}$ 1, pp. 179-255. https://doi.org/10.1002/aris.1440370106.

Brewster, C. (1991). The Management of Expatriates . (Kogan Page, Ed.). London.

Budhwar, P. S., \& Sparrow, P. R. (2002). Strategic HRM through the Cultural Looking Glass: Mapping the Cognition of British and Indian Managers. Organization Studies, vol. 23, $n^{\circ}$ 4, pp. 599-638. https://doi.org/10.1177/0170840602234005.

Caligiuri, P. M. (2000). The Big Five personality characteristics as predictors of expatriate's desire to terminate the assignment and supervisor-rated performance. Personnel Psychology, vol. 53, $\mathrm{n}^{\mathrm{o}}$ 1, pp. 67-88. https://doi.org/10.1111/j.17446570.2000.tb00194.x.

Callon, M., Courtial, J. P., \& Laville, F. (1991). Co-word analysis as a tool for describing the network of interactions between basic and technological research: The case of polymer chemsitry. Scientometrics, vol. 22, $\mathrm{n}^{\mathrm{o}} 1, \quad$ pp. 155-205. https://doi.org/10.1007/BF02019280. 
Cascio, W. F., \& Boudreau, J. W. (2016). The search for global competence: From international HR to talent management. Journal of World Business, vol. 51, $\mathrm{n}^{\circ} 1$, pp. 103-114. https://doi.org/10.1016/j.jwb.2015.10.002.

Cerdin, J. L., \& Bird, A. (2008). Careers in a global context. In T. \& F. In M. M. Harris (Ed.) (Ed.), Handbook of research in international human resource management, pp. 207227. New York.

Cobo, M. J., López-Herrera, A. G., Herrera-Viedma, E., \& Herrera, F. (2011a). An approach for detecting, quantifying, and visualizing the evolution of a research field: A practical application to the Fuzzy Sets Theory field. Journal of Informetrics, vol. 5, n⿳0 1, pp. 146 166. https://doi.org/10.1016/J.JOI.2010.10.002.

Cobo, M. J., López-Herrera, A. G., Herrera-Viedma, E., \& Herrera, F. (2011b). Science mapping software tools: Review, analysis, and cooperative study among tools. Journal of the American Society for Information Science and Technology, vol. 62, $\mathrm{n}^{\circ}$ 7, pp. 1382-1402. https://doi.org/10.1002/asi.21525.

Cobo, M. J., López-Herrera, A. G., Herrera-Viedma, E., \& Herrera, F. (2012). SciMAT: A new science mapping analysis software tool. Journal of the American Society for Information Science and Technology, vol. 63, $\mathrm{n}^{0}$ 8, pp. 1609-1630. https://doi.org/10.1002/asi.22688.

Collings, D. G., Scullion, H., \& Morley, M. J. (2007). Changing patterns of global staffing in the multinational enterprise: Challenges to the conventional expatriate assignment and emerging alternatives. Journal of World Business, vol. 42, $\mathrm{n}^{\circ}$ 2, pp. 198-213. https://doi.org/10.1016/j.jwb.2007.02.005.

Dabic, M., González-Loureiro, M., \& Harvey, M. (2015). Evolving research on expatriates: What is 'known' after four decades (1970-2012). International Journal of Human Resource Management, vol. 26, $\mathrm{n}^{\mathrm{0}} \quad 3, \quad \mathrm{pp}$. 316-337. https://doi.org/10.1080/09585192.2013.845238.

De Cieri, H., Fenwick, M., \& Hutchings, K. (2005). The challenge of international human resource management: Balancing the duality of strategy and practice. International Journal of Human Resource Management, vol. 16, $\mathrm{n}^{\circ}$ 4, pp. 584-598. https://doi.org/10.1080/09585190500051688.

Dickmann, M., Brewster, C., \& Sparrow, P. (2008). International human resource management : a European perspective (2nd ed.). London: Routledge.

Dickmann, M., Suutari, V., Brewster, C., Mäkelä, L., Tanskanen, J., \& Tornikoski, C. (2018). The career competencies of self-initiated and assigned expatriates: assessing the development of career capital over time. The International Journal of Human Resource Management, vol. 29, $\quad \mathrm{n}^{\circ} \quad 16, \quad$ pp. 2353-2371. https://doi.org/10.1080/09585192.2016.1172657.

Dowling, P., \& Schuler, R. S. (1990). International dimensions of human resource management. (PWS-Kent, Ed.). Boston: PWS-Kent.

Farndale, E., Paauwe, J., Morris, S. S., Stahl, G. K., Stiles, P., Trevor, J., \& Wright, P. M. (2010). Context-bound configurations of corporate HR functions in multinational corporations. Human Resource Management, vol. 49, $\mathrm{n}^{\mathrm{o}}$ 1, pp. 45-66. https://doi.org/10.1002/hrm.20333.

Farndale, E., Scullion, H., \& Sparrow, P. (2010). The role of the corporate HR function in global talent management. Journal of World Business, vol. 45, $\mathrm{n}^{0}$ 2, pp. 161-168. https://doi.org/10.1016/j.jwb.2009.09.012.

Guzzo, R. A., Noonan, K. A., \& Elron, E. (1994). Expatriate Managers and the Psychological Contract. Journal of Applied Psychology, vol. 79, $\mathrm{n}^{\circ}$ 4, pp. 617-626. https://doi.org/10.1037/0021-9010.79.4.617. 
Harzing, A. W. (2001). Of bears, bumble-bees, and spiders: The role of expatriates in controlling foreign subsidiaries. Journal of World Business, vol. 36, n 4, pp. 366-379. https://doi.org/10.1016/S1090-9516(01)00061-X.

Hippler, T., Brewster, C., \& Haslberger, A. (2015). The elephant in the room: the role of time in expatriate adjustment. International Journal of Human Resource Management, vol. 26, $\mathrm{n}^{\circ}$ 15, pp. 1920-1935. https://doi.org/10.1080/09585192.2015.1041762.

Hofstede, G. (1980). Culture's consequences : international differences in work-related values. Beverly Hills, CA: Sage Publications.

Hofstede, G. (1993). Cultural constraints in management theories. Academy of Management Perspectives, vol. 7, nº 1, pp. 81-94. https://doi.org/10.5465/ame.1993.9409142061.

Hood, W. W., \& Wilson, C. S. (2001). The literature of bibliometrics, scientometrics, and informetrics. Scientometrics, vol. 52, $\mathrm{n}^{\mathrm{o}}$ 2, pp. 291-314. https://doi.org/10.1023/A:1017919924342.

Javidan, M., House, R. J., Dorfman, P. W., Hanges, P. J., \& De Luque, M. S. (2006). Conceptualizing and measuring cultures and their consequences: A comparative review of GLOBE's and Hofstede's approaches. Journal of International Business Studies, vol. 37, no 6, pp. 897-914. https://doi.org/10.1057/palgrave.jibs.8400234.

Johnson, J. P., Lenartowicz, T., \& Apud, S. (2006). Cross-cultural competence in international business: Toward a definition and a model. Journal of International Business Studies, vol. 37, $n^{\circ}$ 4, pp. 525-543. https://doi.org/10.1057/palgrave.jibs.8400205.

Kirkman, B. L., Lowe, K. B., \& Gibson, C. B. (2006). A quarter century of culture's consequences: A review of empirical research incorporating Hofstede's cultural values framework. Journal of International Business Studies, vol. 37, n 3, pp. 285-320. https://doi.org/10.1057/palgrave.jibs.8400202.

Ko, W. W., \& Liu, G. (2017). Overcoming the liability of smallness by recruiting through networks in China: a guanxi-based social capital perspective. The International Journal of Human Resource Management, vol. 28, $\mathrm{n}^{\circ}$ 11, pp. 1499-1526. https://doi.org/10.1080/09585192.2015.1128467.

Kraimer, M. L., Shaffer, M. A., \& Bolino, M. C. (2009). The influence of expatriate and repatriate experiences on career advancement and repatriate retention. Human Resource Management, vol. 48, $\mathrm{n}^{\circ}$ 1, pp. 27-47. https://doi.org/10.1002/hrm.20265.

Lam, A. (1997). Embedded Firms, Embedded Knowledge: Problems of Collaboration and Knowledge Transfer in Global Cooperative Ventures. Organization Studies, vol. 18, $\mathrm{n}^{\mathrm{o}}$ 6, pp. 973-996. https://doi.org/10.1177/017084069701800604.

Morley, M., \& Heraty, N. (2004). International assignments and global careers. Thunderbird International Business Review, vol. 46, $\mathrm{n}^{\mathrm{0}}$ 6, pp. 633-646. https://doi.org/10.1002/tie.20028.

Morris, S., Snell, S., \& Björkman, I. (2016). An architectural framework for global talent management. Journal of International Business Studies, vol. 47, $\mathrm{n}^{\circ}$ 6, pp. 723-747. https://doi.org/10.1057/jibs.2015.25.

Norris, M., \& Oppenheim, C. (2007). Comparing alternatives to the Web of Science for coverage of the social sciences' literature. Journal of Informetrics, vol. 1, n ${ }^{\circ} 2$, pp. $161-$ 169. https://doi.org/10.1016/J.JOI.2006.12.001.

Ozbilgin, M. (2004). "International” human resource management: Academic parochialism in editorial boards of the "top" 22 journals on international human resource management. In Personnel Review, vol. 33, p. 205-221+265). Emerald Group Publishing Limited. https://doi.org/10.1108/00434804105180559.

Pasamar, S., \& Gallurt, P. (2019). Managing Stress in an International Career. In Managerial Competencies for Multinational Businesses, pp. 44-63. IGI Global. https://doi.org/10.4018/978-1-5225-5781-4.ch003. 
Pritchard, A. (1969). Statistical Bibliography or Bibliometrics. Journal of Documentation, 25, 348-349. - References - Scientific Research Publishing, $n^{\circ}$ 25, pp. 348-349.

Rosenzweig, P. M., \& Nohria, N. (1994). Influences on Human Resource Management Practices in Multinational Corporations. Journal of International Business Studies, vol. 25, no 2, pp. 229-251. https://doi.org/10.2307/155388.

Salamin, X., \& Davoine, E. (2015). International adjustment of female vs male business expatriates. A replication study in Switzerland. Journal of Global Mobility, vol. 3, nº 2, pp. 183-212. https://doi.org/10.1108/JGM-12-2014-0055.

Sambharya, R. B. (1996). Foreign Experience of Top Management Teams and International Diversification Strategies of U.S. Multinational Corporations. Strategic Management Journal, vol. 17, nº 9, pp. 739-746. https://doi.org/10.2307/2486725.

Sánchez, A. D., de la Cruz Del Río Rama, M., \& García, J. Á. (2017). Bibliometric analysis of publications on wine tourism in the databases Scopus and WoS. European Research on Management and Business Economics, vol. 23, $\mathrm{n}^{\mathrm{0}}$ 1, pp. 8-15. https://doi.org/10.1016/J.IEDEEN.2016.02.001.

Santana, M., \& Lopez-Cabrales, A. (2019). Sustainable development and human resource management: A science mapping approach. Corporate Social Responsibility and Environmental Management. https://doi.org/10.1002/csr.1765.

Santana, M., Morales-Sánchez, R., \& Pasamar, S. (2020). Mapping the Link between Corporate Social Responsibility (CSR) and Human Resource Management (HRM): How Is This Relationship Measured? Sustainability, vol. 12, $\mathrm{n}^{\mathrm{o}}$ 4, pp. 1678. https://doi.org/10.3390/su12041678.

Schuler, R. S., Dowling, P. J., \& De Cieri, H. (1993). An integrative framework of strategic international human resource management. Journal of Management, vol. 19, $\mathrm{n}^{\circ} 2$, pp. 419-459. https://doi.org/10.1016/0149-2063(93)90059-v.

Schuler., R. S., Fulkerson, J. R., \& Dowling, P. J. (1991). Strategic performance measurement and management in multinational corporations. Human Resource Management, vol. 30, n 3 3, pp. 365-392. https://doi.org/10.1002/hrm.3930300305.

Selmer, J., \& Leung, A. S. M. (2003). International adjusment of female vs male business expatriates. International Journal of Human Resource Management, vol. 14, $\mathrm{n}^{0}$ 7, pp. 1117-1131. https://doi.org/10.1080/0958519032000114237.

Shaffer, M. A., Gregersen, H., Ferzandi, L. A., Harrison, D. A., \& Black, J. S. (2006). You can take it with you: Individual differences and expatriate effectiveness. Journal of Applied Psychology, vol. 91, no 1, pp. 109-125. https://doi.org/10.1037/0021-9010.91.1.109.

Shaffer, M. A., \& Harrison, D. A. (1998). Expatriates' Psychological Withdrawal From International Assignments: Work, Nonwork, And Family Influences. Personnel Psychology, vol. 51, $\mathrm{n}^{\mathrm{o}}$ 1, pp. 87-118. https://doi.org/10.1111/j.17446570.1998.tb00717.x.

Shaffer, M. A., Harrison, D. A., \& Gilley, K. M. (1999). Dimensions, Determinants, and Differences in the Expatriate Adjustment Process. Journal of International Business Studies. Palgrave Macmillan Journals. https://doi.org/10.2307/155465.

Shaffer, M. A., Kraimer, M. L., Chen, Y.-P., \& Bolino, M. C. (2012). Choices, Challenges, and Career Consequences of Global Work Experiences. Journal of Management, vol. 38, $\mathrm{n}^{\mathrm{o}}$ 4, pp. 1282-1327. https://doi.org/10.1177/0149206312441834.

Shen, J., \& Jiang, F. (2015). Factors influencing Chinese female expatriates' performance in international assignments. International Journal of Human Resource Management, vol. 26, $\mathrm{n}^{\circ}$ 3, pp. 299-315. https://doi.org/10.1080/09585192.2011.581637.

Small, H. (1999). Visualizing science by citation mapping. Journal of the American Society for Information Science, vol. 50, $\mathrm{n}^{\circ}$ 9, pp. 799-813. https://doi.org/10.1002/(SICI)10974571(1999)50:9<799::AID-ASI9>3.0.CO;2-G. 
Stahl, G. K., Miller, E. L., \& Tung, R. L. (2002). Toward the boundaryless career: A closer look at the expatriate career concept and the perceived implications of an international assignment. Journal of World Business, vol. 37, $\mathrm{n}^{\circ}$ 3, pp. 216-227. https://doi.org/10.1016/S1090-9516(02)00080-9.

Sullivan, S. E., \& Baruch, Y. (2009). Advances in Career Theory and Research: A Critical Review and Agenda for Future Exploration. Journal of Management, vol. 35, $\mathrm{n}^{\circ}$ 6, pp. 1542-1571. https://doi.org/10.1177/0149206309350082.

Tarique, I., \& Schuler, R. S. (2010). Global talent management: Literature review, integrative framework, and suggestions for further research. Journal of World Business, vol. 45, $\mathrm{n}^{\mathrm{o}}$ 2, pp. 122-133. https://doi.org/10.1016/J.JWB.2009.09.019.

Taylor, S., Beechler, S., \& Napier, N. (1996). Toward an Integrative Model of Strategic International Human Resource Management. The Academy of Management Review, vol. 21, no 4, pp. 959. https://doi.org/10.2307/259160.

Tung, R. L. (1998). American expatriates abroad: From neophytes to cosmopolitans. Journal of World Business, vol. 33, $\mathrm{n}^{\circ}$ 2, pp. 125-144. https://doi.org/10.1016/S10909516(98)90002-5.

Werner, S. (2002). Recent Developments in International Management Research: A Review of 20 Top Management Journals. Journal of Management, vol. 28, $\mathrm{n}^{\circ}$ 3, pp. 277-305. https://doi.org/10.1177/014920630202800303.

Zhong, W., \& Chin, T. (2015). The role of translation in cross-cultural knowledge transfer within a MNE's business networks: A 3D-hierarchical model in China. Chinese Management Studies, vol, 9, $\mathrm{n}^{\circ}$ 4, pp. 589-610. https://doi.org/10.1108/CMS-06-20150114. 